\title{
Bruchpilot and Synaptotagmin collaborate to drive rapid glutamate release and active zone differentiation
}

\section{Mila M. Paul ${ }^{1}$, Martin Pauli ${ }^{1}$, Nadine Ehmann ${ }^{1}$, Stefan Hallermann ${ }^{2}$, Markus Sauer ${ }^{3}$, Robert J. Kittel $^{1}$ * and Manfred Heckmann ${ }^{1}$ *}

${ }^{1}$ Department of Neurophysiology, Institute of Physiology, Julius-Maximilians-University Würzburg, Würzburg, Germany

${ }^{2}$ Carl-Ludwig-Institute for Physiology, University of Leipzig, Leipzig, Germany

${ }^{3}$ Department of Biotechnology and Biophysics, Julius-Maximilians-University Würzburg, Würzburg, Germany

Edited by:

Christian D. Wilms, University College London, UK

\section{Reviewed by:}

Annalisa Scimemi, State University of New York Albany, USA

Dion Dickman, University of Southern California, USA

\section{${ }^{*}$ Correspondence:}

Manfred Heckmann and Robert J.

Kittel, Department of

Neurophysiology, Institute of

Physiology, Julius-Maximilians-

University Würzburg, Röntgenring 9,

97070 Würzburg, Germany

e-mail: heckmann@uni-wuerzburg.de;

robert.kitte@@uni-wuerzburg.de
The active zone (AZ) protein Bruchpilot (Brp) is essential for rapid glutamate release at Drosophila melanogaster neuromuscular junctions (NMJs). Quantal time course and measurements of action potential-waveform suggest that presynaptic fusion mechanisms are altered in brp null mutants $\left(b r p^{69}\right)$. This could account for their increased evoked excitatory postsynaptic current (EPSC) delay and rise time (by about $1 \mathrm{~ms}$ ). To test the mechanism of release protraction at $b_{r p} 69 \mathrm{AZs}$, we performed knock-down of Synaptotagmin-1 (Syt) via RNAi $\left(s y t^{K D}\right.$ ) in wildtype (wt), brp ${ }^{69}$ and rab3 null mutants (rab3 $3^{\text {rup }}$ ), where Brp is concentrated at a small number of AZs. At wt and rab3 ${ }^{\text {rup }}$ synapses, syt ${ }^{K D}$ lowered EPSC amplitude while increasing rise time and delay, consistent with the role of Syt as a release sensor. In contrast, syt ${ }^{K D}$ did not alter EPSC amplitude at brp ${ }^{69}$ synapses, but shortened delay and rise time. In fact, following $s y t^{K D}$, these kinetic properties were strikingly similar in wt and brp 69 , which supports the notion that Syt protracts release at $b r p^{69}$ synapses. To gain insight into this surprising role of Syt at brp ${ }^{69} \mathrm{AZs}$, we analyzed the structural and functional differentiation of synaptic boutons at the NMJ. At 'tonic' type Ib motor neurons, distal boutons contain more AZs, more Brp proteins per AZ and show elevated and accelerated glutamate release compared to proximal boutons. The functional differentiation between proximal and distal boutons is Brp-dependent and reduced after $s y t^{K D}$. Notably, syt ${ }^{K D}$ boutons are smaller, contain fewer Brp positive AZs and these are of similar number in proximal and distal boutons. In addition, super-resolution imaging via $d S T O R M$ revealed that $s y t^{K D}$ increases the number and alters the spatial distribution of Brp molecules at AZs, while the gradient of Brp proteins per AZ is diminished. In summary, these data demonstrate that normal structural and functional differentiation of Drosophila AZs requires concerted action of Brp and Syt.

Keywords: Bruchpilot, active zone, neurotransmitter release, synaptic delay, presynaptic differentiation, synaptotagmin, $d$ STORM

\section{INTRODUCTION}

Active zones (AZs) allow exquisite spatial and temporal control of vesicle fusion. Large multidomain proteins rich in coiled-coil sequences such as Bassoon, Piccolo and the CAST/ERC family member Brp are major structural and functional organizers of AZs (Südhof, 2012). Their abundance appears to correlate positively with neurotransmitter release (Graf et al., 2009; Matz etal., 2010; Weyhersmüller etal., 2011; Ehmann etal., 2014; Peled et al., 2014).

At Drosophila melanogaster NMJs, Brp is crucial for synchronous glutamate release and the clustering of calcium channels at AZs (Kittel etal., 2006; Wagh etal., 2006). Linking the amount of Brp or Bassoon per AZ to the number and spatial arrangement of calcium channels may account for the correlation with release probability, e.g., in the context of synaptic homeostasis (Matz et al., 2010; Weyhersmüller et al., 2011; Ehmann etal., 2014). Slight increases in coupling distance in the $20-40 \mathrm{~nm}$ range reduce release probability dramatically while changing kinetic release parameters to a lesser extent (Neher, 1998; Eggermann etal., 2011; Schmidt etal., 2013; Vyleta and Jonas, 2014). Differences in coupling distance are therefore ideal for scaling the amount of release, whereas controlling its time course appears to require additional molecular mechanisms.

The main kinetic transmitter release parameters are synaptic delay, rise, and decay times. In the present study, we focus on synaptic delay and EPSC rise time. Notably, the latter is increased by more than $1 \mathrm{~ms}$ at synapses lacking Brp, while release probability drops by comparison only moderately (Kittel et al., 2006; Eggermann et al., 2011). This marked kinetic change appears disproportional to the reduction in release probability. While synaptic delay has not yet been analyzed at $b r p^{69}$ synapses, it is usually fairly constant for a wide range of release probabilities (Barrett and Stevens, 1972; Datyner and Gage, 1980).

While the molecular mechanisms controlling release kinetics are complex and not well understood (Neher, 2010), it is 
clear that the vesicle protein Syt plays an important role (Brose et al., 2002; Young and Neher, 2009). As initially suggested more than 20 years ago (DiAntonio et al., 1993; Littleton et al., 1993; Geppert etal., 1994), Syt is crucial for triggering release and may act both as a calcium sensor and a vesicle fusion clamp. In fact, its role may change from clamp to sensor upon calcium influx into the presynaptic terminal (DeBello et al., 1993; Walter et al., 2011).

To clarify the molecular mechanisms that shape the time course of release we analyzed the interaction between Brp and Syt. We find that in addition to prolonged EPSC rise time, synaptic delay is strongly increased at $b r p^{69}$ synapses. Interestingly, whereas Syt is necessary for the increase in both kinetic parameters, it has little effect on the amount of transmitter released from $b r p^{69}$ AZs. Following up on the functional interaction of Brp and Syt, our data suggest central roles of these two proteins in the spatial differentiation of AZs and reveal that the number of AZs per bouton, as well as the number and distribution of Brp molecules per AZ is Syt-dependent.

\section{MATERIALS AND METHODS FLY STOCKS}

Drosophila larvae were raised in vials on standard corn meal (Ashburner, 1989) at $25^{\circ} \mathrm{C}$ for focal recordings in Figure $\mathbf{1}$ (wt and $b r p^{69}$ ) or at $29^{\circ} \mathrm{C}$ for reliable RNAi expression in all other experiments (mutant and control groups). For Syt RNAi, we expressed syt1_RNAi ${ }^{8875}$ Vienna Drosophila Resource Center (VDRC) specifically in motor neurons (ok6-GAL4/+; UAS-syt1_RNAi $i^{8875} /+$ ) or panneuronally (elav-GAL4/UAS-syt1_RNAi ${ }^{8875}$ ) using the binary UAS-GAL4 expression system (Brand and Perrimon, 1993). $b r p^{69}$ and rab3 ${ }^{r u p}$ were used as previously described (Kittel etal., 2006; Graf etal., 2009). To combine $b r p^{69}$ and rab3 $3^{\text {rup }}$ with syt1_RNAi ${ }^{8875}$ the following lines were generated: brp ${ }^{69}$ ok6-GAL4/ $d f(2 R) B S C 29 ; \quad U A S$-syt1_RNAi $i^{8875} /+$ and $b r p^{69} / d f(2 R) B S C 29 ;$ elav-GAL4/UAS-syt1_RNAi ${ }^{8875}$ (brp ${ }^{69}$, syt $\left.t^{K D}\right) ; r a b 3^{\text {rup }} / d f(2 R) E D 2076$ ok6-GAL4; UAS-syt1_RNAi ${ }^{8875} /+$ and rab3 ${ }^{\text {rup }} / d f(2 R) E D 2076$; elav-GAL4/UAS-syt1_RNAi ${ }^{8875}$ (rab3 $^{\text {rup }}$, syt $\left.{ }^{K D}\right)$; ok6-GAL4/+ and elav-GAL4/+ served as wt controls. For focal recordings, GFP was expressed in presynaptic terminals: ok6-GAL4/+; UAS-CD8::GFP/+ (wt), brp ${ }^{69}$ ok6GAL4/df(2R)BSC29;UAS-CD8::GFP/+ (brp $\left.{ }^{69}\right)$ and ok6-GAL4/+; UAS-CD8::GFP/UAS-Syt1_RNAi $i^{8875}\left(s y t^{K D}\right)$. For non-allelic noncomplementation the following genotypes were used: $w^{1118}(\mathrm{wt})$, $s y t 1^{A D 4} /+$ (DiAntonio et al., 1993), brp ${ }^{69} /+$ (Kittel et al., 2006) and $s y t 1^{A D 4} / b r p^{69}\left(s y t^{A D 4}+/+b r p^{69}\right)$.

\section{PHYSIOLOGICAL SOLUTION AND PREPARATION}

The composition of the extracellular, physiological, hemolymphlike saline (HL-3, Stewart et al., 1994) was (in mM): $\mathrm{NaCl} 70, \mathrm{KCl}$, $\mathrm{MgCl}_{2}$ 20, $\mathrm{NaHCO}_{3}$ 10, trehalose 5, sucrose 115, HEPES 5, $\mathrm{CaCl}_{2}$ as indicated, $\mathrm{pH}$ adjusted to 7.2. Wandering male third instar larvae were dissected in $\mathrm{HL}-3$ without $\mathrm{CaCl}_{2}$. All experiments were carried out at NMJs formed on ventral abdominal muscles 6/7 in segments A2 and A3.

\section{FOCAL RECORDINGS}

Macropatch recordings in Figure 1 were performed in HL-3 saline containing $1 \mathrm{mM}\left[\mathrm{Ca}^{2+}\right]_{\mathrm{Ex}}$ essentially as reported previously (Pawlu etal., 2004). Bath temperature was kept constant at $18 \pm 0.5^{\circ} \mathrm{C}$ using a Peltier element (27 W, Conrad Electronic) glued to the bath inflow with heat-conductive paste (Fischer Elektronik). EPSCs were elicited using a $0.2 \mathrm{~Hz}$ nerve-stimulation protocol with $0.2 \mathrm{~ms}$ pulse duration and amplitudes slightly above the threshold for eliciting an action potential via a suction electrode (filled with extracellular solution). Recording electrodes with openings of about 5-10 $\mu \mathrm{m}$ diameter below the tip had resistances of $250 \mathrm{k} \Omega$ when filled with HL-3. About 20 EPSCs were recorded per site and analyzed with IgorPro 5.04 (Wavemetrics). The data were digitally filtered at $3 \mathrm{kHz}$ (Gaussian filter), baseline subtracted and the average of all failures was
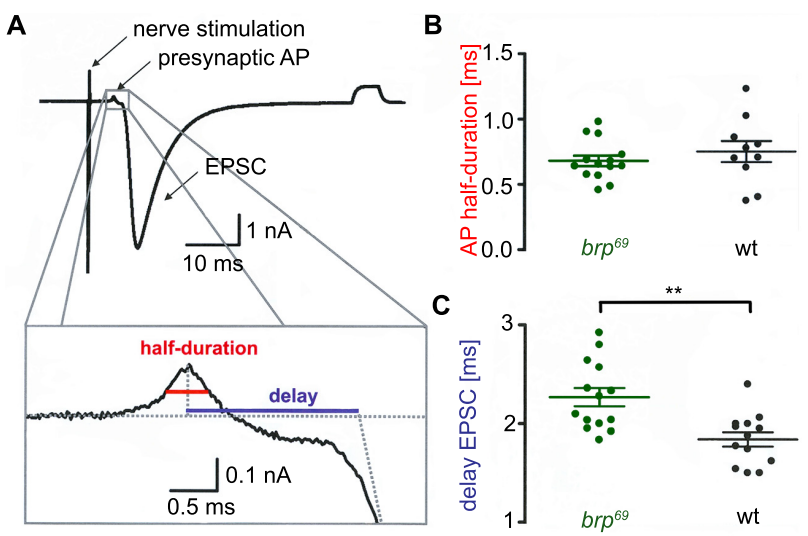

FIGURE 1 | Presynaptic action potential and synaptic delay in $b_{r p}^{69}$. (A) Focal recording from a Drosophila wt NMJ. Nerve stimulation (left arrow) elicited an action potential in the suction electrode which traveled to the presynaptic bouton under the focal electrode and lead to deflections (presynaptic AP, second arrow) prior to the large compound EPSC (third arrow). A subthreshold pulse through the focal electrode elicited the final upward deflection (right side of the trace and allowed to measure electrode and seal resistance). The lower panel shows the enlarged presynaptic AP and illustrates how half-duration of the positive AP deflection (red) and synaptic delay (blue) were determined. (B) While AP half-duration is unchanged between $b_{r p}{ }^{69}$ (green) and wt (black), (C) synaptic delay is prolonged in brp 69 . Shown are single values (dots) and mean \pm SD. 
subtracted from the currents. AP durations were measured at half amplitude of the positive deflection (Dudel, 1965) and synaptic delay was measured from the peak of the AP to the point at which the back extrapolation of the EPSC current rising phase crossed the baseline (Figure 1). Focal recordings in Figure 6 were performed in HL-3 saline containing $0.5 \mathrm{mM}\left[\mathrm{Ca}^{2+}\right]_{\mathrm{Ex}}$. Bath temperature was kept constant at $20 \pm 1^{\circ} \mathrm{C}$. Focal electrodes (resistances $600 \pm 50 \mathrm{k} \Omega$ when filled with HL-3 solution) were positioned on proximal or distal type Ib boutons of muscles 6/7. EPSCs were elicited using a $0.2 \mathrm{~Hz}$ nerve-stimulation protocol with $0.2 \mathrm{~ms}$ pulse duration and $7 \mathrm{~V}$ amplitude. Traces were low-pass filtered at $20 \mathrm{kHz}$, and recorded and stored with Patchmaster using an EPC10 double patch clamp amplifier (HEKA electronics). About 60 EPSCs were averaged per site and analyzed with Igor Pro 6.05 (Wavemetrics). 10 mg EGTA-AM (membrane permeable tetraacetoxymethyl ester of ehtyleneglycol-bis(Baminoethyl)-N,N,N',N'-tetraacetic acid, Calbiochem Germany) was dissolved in DMSO with 20\% Pluronic (Invitrogen) to obtain a stock solution of $10 \mathrm{mM}$ EGTA. This stock solution was diluted 1:100 with calcium-free HL-3 and applied to the dissected preparation for $10 \mathrm{~min}$. After incubation preparations were washed for 5 minutes with HL-3 (Müller et al., 2012) and recordings were performed in HL-3 containing $1.0 \mathrm{mM}\left[\mathrm{Ca}^{2+}\right]_{\mathrm{Ex}}$ as described above.

\section{TWO-ELECTRODE VOLTAGE CLAMP RECORDINGS (TEVC)}

Two-electrode voltage clamp-recordings (Figure 3) were performed essentially as previously described (Kittel etal., 2006) using an Axo Clamp 2B amplifier (Axon Instruments, Molecular Devices). All measurements were made from muscle 6 at $21 \pm 1^{\circ} \mathrm{C}$ bath temperature. Intracellular electrodes were filled with $3 \mathrm{M} \mathrm{KCl}$ and had resistances of $12-15 \mathrm{M} \Omega$. $\mathrm{V}_{\text {holding }}$ was $-60 \mathrm{mV}$ for evoked EPSCs. Only cells with an initial membrane potential of at least $-50 \mathrm{mV}$ and $\geq 4 \mathrm{M} \Omega$ input resistance were analyzed. Synaptic responses were generated by pulses of $0.3 \mathrm{~ms}$ length and $5-10 \mathrm{~V}$ amplitude, applied via a suction electrode (filled with extracellular solution) and low-pass filtered at $10 \mathrm{kHz}$. We applied a $0.2 \mathrm{~Hz}$ stimulation protocol, averaged 20 EPSCs per muscle cell and analyzed the data with Clampfit (Axon Instruments, Molecular Devices).

\section{IMAGING}

Larvae were dissected in ice-cold HL-3 standard saline without $\mathrm{CaCl}_{2}$, fixed with $4 \%$ paraformaldehyde in $0.1 \mathrm{M}$ phosphate buffered saline (PBS) for $10 \mathrm{~min}$ and blocked with PBT (PBS containing $0.05 \%$ Triton X-100, Sigma) including 5\% natural goat serum (Dianova) for $30 \mathrm{~min}$. Primary antibodies were added for overnight staining at $4^{\circ} \mathrm{C}$. After three washing steps with PBS (20 min each), preparations were incubated with secondary antibodies for $2-4 \mathrm{~h}$ at room temperature followed by three washing steps with PBS. Filets were mounted using Vectashield (Vector Laboratories) and images were aquired using an Apotome System (Zeiss, Axiovert 200M Zeiss, objective $63 \mathrm{x}$, NA 1.4, oil). Antibodies were used at the following concentrations: mouse monoclonal antibody $(\mathrm{mAb}) \mathrm{Brp}^{\mathrm{Nc} 82}$ (1:250), Alexa Fluor 488-conjugated goat $\alpha$-mouse (Invitrogen) and Cy3-conjugated goat $\alpha$-horseradish peroxidase (HRP,
Jackson Immuno Research) antibodies (1:250), rabbit $\alpha$-DsytCL1 (Mackler et al., 2002) and Cy3-conjugated goat $\alpha$-rabbit (Jackson Immuno Research, 1:250) antibodies. Z-stacks of 1520 single images taken every $250 \mathrm{~nm}$ were maximum projected and analyzed in Image $(1.440, \mathrm{NIH})$. Brp puncta per NMJ and per bouton were quantified manually. Using the three terminal boutons of type $\mathrm{Ib}$ and Is branches the respective structural gradient was analyzed. Distal boutons were located at the end of bouton chains, while proximal boutons were closer to the entry site of the motor neuron. Bouton area, length (along chain axis) and width ( $90^{\circ}$ to length) were measured using $\alpha$-HRP stainings.

\section{dSTORM (DIRECT STOCHASTIC OPTICAL RECONSTRUCTION MICROSCOPY)}

Larvae were dissected, fixed and washed as described above and super-resolution imaging was performed essentially as previously reported (Ehmann et al., 2014). Preparations were incubated with $\mathrm{mAb} \operatorname{Brp}^{N c 82}$ (1:2000) and secondary antibody goat $\alpha$-mouse $\mathrm{F}(\mathrm{ab})_{2}$ fragments (A10534, Invitrogen) labeled with Cy5-NHS (PA15101, GE Healthcare) at a concentration of $5.2 \times 10^{-8} \mathrm{M}$ or with rabbit $\alpha$-Dsyt-CL1 (1:10000, Mackler et al., 2002) and secondary antibody goat $\alpha$-rabbit $\mathrm{F}\left(\mathrm{ab}^{\prime}\right)_{2}$ fragments labeled with Alexa Fluor 647 (1:500, Invitrogen). Boutons were visualized with Alexa Fluor 488 conjugated goat $\alpha$-horseradish-peroxidase antibody ( $\alpha$-HRP, 1:250, Jackson Immuno Research). After staining, larval preparations were incubated in $100 \mathrm{mM}$ Mercaptoethylamin (MEA, Sigma-Aldrich) buffer in PBS, pH 7.8-7.9 to allow reversible switching of single fluorophores during data acquisiton (van de Linde et al., 2008). Images were acquired using an inverted microscope (Olympus IX-71, 60x, NA 1.45, oil immersion) equiped with a nosepiece-stage (IX2-NPS, Olympus). $644 \mathrm{~nm}$ (iBEAM-SMART-640-S, Toptica), and $488 \mathrm{~nm}$ (iBEAM-SMART-488-S, Toptica) lasers were used for excitation of Cy5 and Alexa Fluor488, respectively. Laser beams were passed through a clean-up filter (Brightline HC 642/10, Semrock, and ZET 488/10, Chroma, respectively) and two dichroic mirrors (Laser-MUX BS 514-543 and HC-quadband BP, Semrock) onto the probe. The emmitted fluorescence was filtered with a quadband-filter (HC-quadband 446/523/600/677, Semrock) and divided onto two cameras (iXon Ultra DU-897-U, Andor) using a dichroic mirror (HC-BS 640 imaging, Semrock). In addition, fluorescence was filtered using a longpass(Edge Basic 635, Semrock) or bandpass-filter (Brightline HC $525 / 50$, Semrock) for red and green channels, respectively. Pixel sizes were $126 \mathrm{~nm}$ (red) and $128 \mathrm{~nm}$ (green). Single fluorophores were localized and high resolution-images were reconstructed with rapidSTORM (Heilemann et al., 2008; Wolter etal., 2010; van de Linde etal., 2011; Wolter etal., 2012; www.super-resolution.de). Only fluorescence spots with more than 1000 photons were analyzed $(10 \mathrm{~nm} /$ pixel sub-pixel binning). Data were processed with ImageJ (1.440, NIH), AZ area and Brp localizations per AZ were analyzed as previously described Ehmann etal. (2014). AZs were assigned to single boutons using the $\alpha$-HRP signal. For Syt1 quantification (Figure 2) we determined localization counts in single type Ib boutons that were defined according to the $\alpha$-HRP signal. 


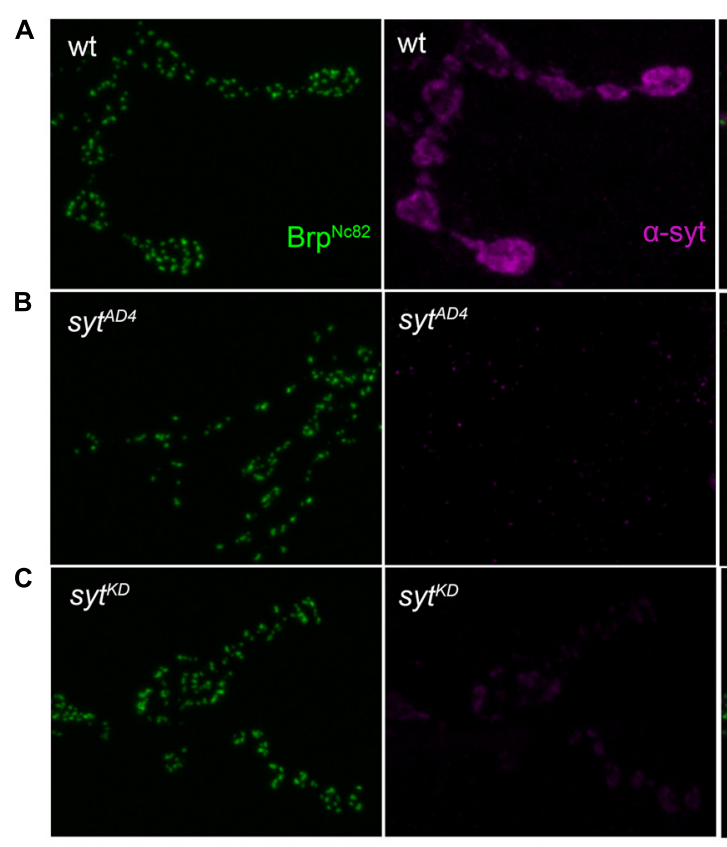

D
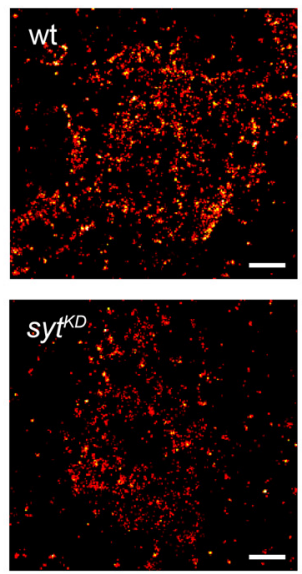

E

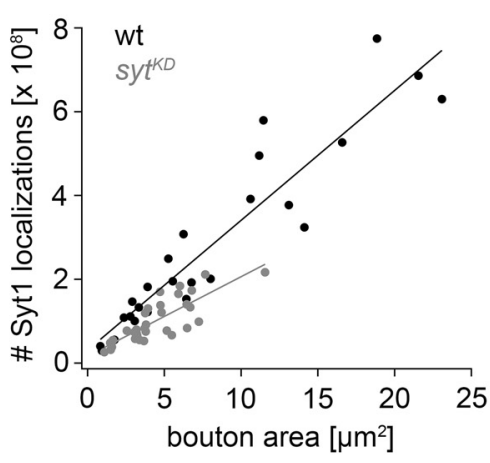

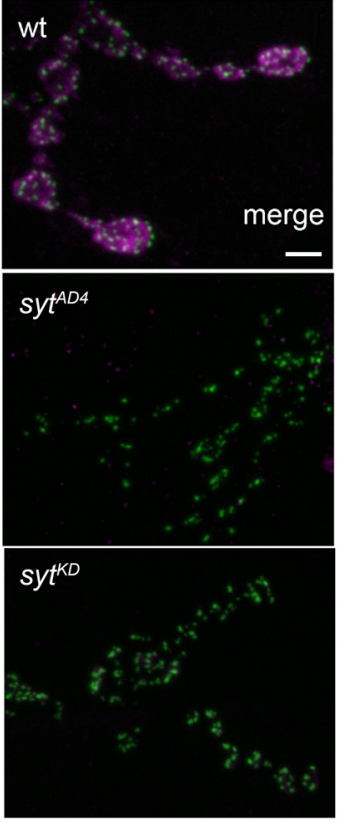

$\mathbf{F}$

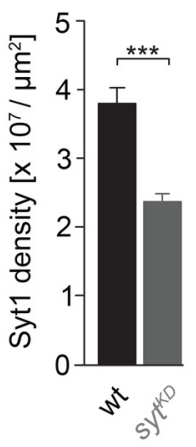

FIGURE 2 | Ribonucleic acid interference decreases presynaptic Syt protein levels. (A) Drosophila wt NMJ on larval abdominal muscles 6/7 stained with antibodies against $\operatorname{Brp}\left(\operatorname{Brp}^{N c 82}\right.$, green) and Synaptotagmin ( $\alpha$-Syt, magenta). Brp demarks individual AZs, whereas Syt labels synaptic vesicles predominately distributed around the bouton circumference. (B) The $\alpha$-Syt signal is absent in Syt null mutans $\left(\right.$ syt $\left.^{A D 4}\right)$ and $(\mathbf{C})$ strongly reduced after Syt knock-down (syt ${ }^{K D}$ ) through presynaptically (ok6-GAL4) driven RNA interference (UAS-syt1-RNA;8875). (D) dSTORM images of single presynaptic boutons stained against Syt1. (E) Number of single-fluorophore localization events by $d$ STORM. Syt1 localizations plotted against bouton area for wt (black) and syt ${ }^{K D}$ (gray) with respective regression lines. Dots represent values for single boutons. (F) Summary bar graph indicates the decrease of Syt1 density in boutons of similar size (below $10 \mu \mathrm{m}^{2}$ ) to $62.38 \%$ in syt ${ }^{K D}$ compared to wt $(p<0.001)$. Scale bar $=2 \mu \mathrm{m}(\mathbf{A}-\mathbf{C})$ and $300 \mathrm{~nm}$ (D).
Localization densities were analyzed only in boutons with areas $<10 \mu \mathrm{m}$.

\section{STATISTICAL ANALYSIS}

Statistical analyses were performed with Sigma Plot 12 (Systat Software) using the non-parametric Mann-Whitney rank sum test. Linear fits for mean \pm SEM Brp localizations per AZ (Figure 9) were made in Igor Pro (Wavemetrics) and statistical analysis was performed using the non-parametric Spearman correlation coefficient. Asterisks indicate the significance level ${ }^{*} p<0.05$, $\left.{ }^{* *} p<0.01,{ }^{* * *} p<0.001\right)$. Data are reported as mean \pm SEM unless indicated otherwise and $n$ denotes sample number.

\section{RESULTS}

\section{SYNAPTIC DELAY IS INCREASED IN brp $^{69}$}

We performed focal recordings using macropatch electrodes which allow simultaneous measurements of presynaptic action potentials (AP) and synaptic release (Dudel, 1965). EPSCs evoked by $0.2 \mathrm{~Hz}$ nerve stimulation were recorded at $b r p^{69}$ and wt larval NMJs on muscles $6 / 7$ to measure half-duration of the positive AP deflection and synaptic delay (Figure 1A). Whereas AP wave form was unchanged in $b r p^{69}$ compared to wt, synaptic delay was significantly increased $(2.27 \pm 0.3 \mathrm{~ms}$ and $1.8 \pm 0.3 \mathrm{~ms}$ mean $\pm \mathrm{SD}$, $p=0.0014, n=14$, and 13 for $b r p^{69}$ and wt, Figures 1B,C). As quantal time course is normal in $b r p^{69}$ (Kittel et al., 2006), 
the increase in release kinetics at $b r p^{69}$ synapses is likely due to alterations in presynaptic fusion mechanisms.

\section{IMPACT OF SYT ON DIFFERENT AZ STATES}

We used wt, $b r p^{69}$ and $r a b 3^{r u p}$ to define explicit AZ conditions: (i) normal organization, (ii) disorganized lacking Brp (Kittel et al., 2006), and (iii) large accumulation of Brp proteins (Graf et al., 2009; Ehmann et al., 2014), respectively. To determine the impact of the putative calcium sensor Syt on synchronous transmitter release in the context of different AZ states we combined these genotypes with $s y t^{K D}$. Protein levels of endogeneous Syt were decreased via RNAi (syt1-RNAi ${ }^{8875}$, see experimental procedures). By engaging the binary UAS-Gal4 expression system (Brand and Perrimon, 1993), syt1-RNAi was driven in larval glutamatergic motor neurons or panneuronally. To confirm that presynaptic Syt expression was reduced by this strategy, immunostainings of larval NMJs were performed using an antiserum against Syt1 (Mackler et al., 2002; Figure 2). Whereas presynaptic terminals of $s y t^{A D 4}$ were completely devoid of Syt, there was residual though heavily reduced protein expression in $s y t^{K D}$ compared to wt (Figure 2C). Furthermore, we quantified the protein reduction following Syt knock-down with dSTORM (Ehmann et al., 2014). Comparison of Syt1 localization numbers in boutons of similar size in wt and $s y t^{K D}$ revealed a reduction to $62.38 \%$ in $s y t^{K D}$ (Figures 2D-F). To address the functional consequences of $s y t^{K D}$ at wt, $b r p^{69}$, and $r a b 3^{r u p}$ synapses, postsynaptic currents in response to low-frequency nerve stimulation were recorded in two-electrode voltage clamp mode (TEVC) from larval ventral abdominal muscles 6/7 (Figure 3). Both panneuronal (Figure 3) and motoneuronal (data not shown) RNAi expression gave essentially comparable results. At wt synapses, $s y t^{K D}$ decreased EPSC amplitude and lengthened rise time (amplitude: $30.0 \pm 4.5 \mathrm{nA}$ and $53.6 \pm 4.7 \mathrm{nA}, p=0.002$; rt: $1.3 \pm 0.1 \mathrm{~ms}$ and $1.0 \pm 0.03 \mathrm{~ms}$, $p<0.001 ; n=11$ and 17 for $s y t^{K D}$ and wt, Figure 3B) consistent with the role of Syt as a sensor for fast release (DiAntonio et al., 1993; Littleton et al., 1993). Similarly, at rab3 ${ }^{\text {rup }}$ synapses, syt ${ }^{K D}$ reduced the amplitude and increased the delay of postsynaptic responses (amplitude: $19.5 \pm 2.1 \mathrm{nA}$ and $50.3 \pm 5.3 \mathrm{nA}, p<0.001$; delay: $1.7 \pm 0.1 \mathrm{~ms}$ and $1.4 \pm 0.04 \mathrm{~ms}, p=0.013 ; n=14$ and 10 for $r a b 3^{r u p}, s y t^{K D}$, and $\left.r a b 3^{\text {rup }}\right)$. Strikingly, syt $t^{K D}$ at $b r p^{69}$ synapses left current amplitudes unchanged $(35.1 \pm 6.7 \mathrm{nA}$ and $24.8 \pm 3.3 \mathrm{nA}$, $p>0.05)$ and in fact accelerated EPSC rise time and delay (rt: $1.4 \pm 0.1 \mathrm{~ms}$ and $2.2 \pm 0.2 \mathrm{~ms}, p=0.008$; delay: $1.6 \pm 0.03 \mathrm{~ms}$ and $1.9 \pm 0.1 \mathrm{~ms}, p<0.001 ; n=12$ and 11 for $b r p^{69}$, syt $t^{K D}$, and $\left.b r p^{69}\right)$. These results illustrate that Syt is necessary for efficient and rapid vesicle fusion at AZs with normal or increased Brp levels. In contrast, vesicle release from $b r p^{69}$ AZs appears less dependent on Syt. We did not find any changes in size or kinetics of quantal events in $b r p^{69}$ and $b r p^{69}$, syt $t^{K D}$ that could explain these effects (amplitude: $0.89 \pm 0.04 \mathrm{nA}$ and $0.90 \pm 0.04 \mathrm{nA}$; $\mathrm{rt}: 1.0 \pm 0.06 \mathrm{~ms}$ and $1.0 \pm 0.04 \mathrm{~ms}$; tau: $6.02 \pm 0.6 \mathrm{~ms}$ and $7.2 \pm 0.4 \mathrm{~ms}$; all $p>0.05 ; n=10$ and 14 for $b r p^{69}$ and $b r p^{69}$, syt $t^{K D}$, respectively). Thus, the changes in release kinetics following $s y t^{K D}$ suggest that Syt protracts release at $b r p^{69}$ AZs.

\section{REDUCED EGTA SENSITIVITY IN $b r p^{69}$, syt $^{K D}$}

To further clarify how $s y t^{K D}$ affects release we tested the influence of EGTA-AM in $s y t^{K D}$ and $b r p^{69}, s y t^{K D}$ in focal recordings

\section{A}
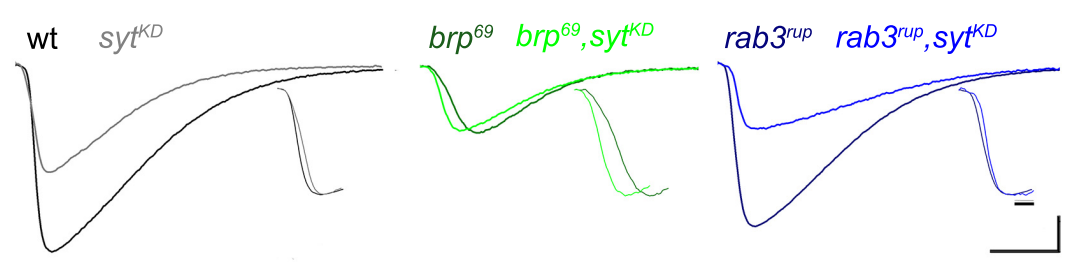

B
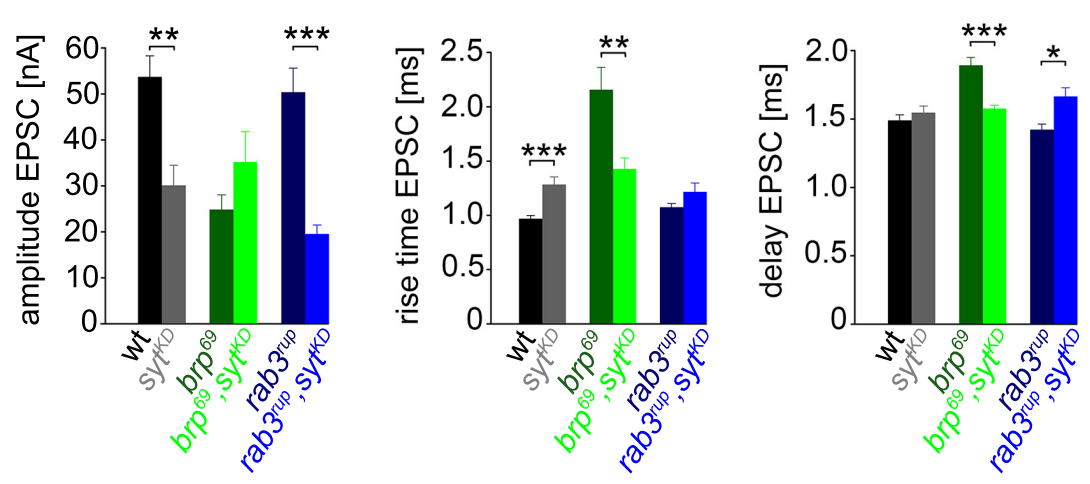

FIGURE 3 | Effect of $s y t^{K D}$ on amplitude and kinetics of the postsynaptic response. (A) Two-electrode voltage clamp recordings in physiological solution with $1.0 \mathrm{mM}\left[\mathrm{Ca}^{2+}\right]_{\text {Ex }}$ and $0.2 \mathrm{~Hz}$ nerve stimulation. Traces from wt (black), brp 69 (dark green), and rab3 $3^{\text {rup }}$ (dark blue) are shown superimposed with recordings of the respective genotypes after syt ${ }^{K D}$ (brighter shades). Insets show normalized events to highlight the differences in synaptic delay and postsynaptic current rise time. Scale bars $=5 \mathrm{~ms}, 10 \mathrm{nA}$ (main traces), and $1 \mathrm{~ms}$ (insets). (B) Summary bar graphs for mean \pm SEM amplitude, current rise time and synaptic delay for all six genotypes, color coded as in (A). 
(Figure 4, Kittel et al., 2006). Consistent with earlier work (Maximov and Südhof, 2005) release in $s y t^{K D}$ was significantly reduced $(0.37 \pm 0.03 \mathrm{nA}$ and $0.22 \pm 0.03 \mathrm{nA}, p=0.002, n=16$ each), whereas the reduction was not significant in $b r p^{69}, s y t^{K D}$ $(0.28 \pm 0.05 \mathrm{nA}$ and $0.21 \pm 0.03 \mathrm{nA}, p>0.05, n=17$, and 10 without and with EGTA). This is in contrast to the findings described in Kittel et al. (2006) for $b r p^{69}$ and suggests that Syt knock-down reduces coupling distance in $b r p^{69}$ and that Syt's role in positional priming (Young and Neher, 2009) requires Brp.

\section{BRP IS DISTRIBUTED UNEVENLY AT WT AND rab3 ${ }^{\text {rup }}$ NMJs}

The innervation of ventral abdominal muscles $6 / 7$ is shared between two functionally distinct motoneurons in Drosophila larvae (Karunanithi et al., 2002): the MN6/7b-Ib (RP3) neuron gives rise to large type Ib boutons (Atwood et al., 1993), whereas the MNSNb/d-Is neuron forms smaller type Is boutons (Kurdyak et al., 1994; Lnenicka and Keshishian, 2000; Hoang and Chiba, 2001). We performed immunostainings and counted the number of Brp puncta per bouton as an estimate of the number of AZs. In addition, staining against horseradish-peroxidase (HRP) was used to measure dimensions of presynaptic arborizations (Jan and Jan, 1982; Figure 5). We found an uneven Brp distribution in wt type Ib motoneurons with more Brp positive AZs in distal than

A

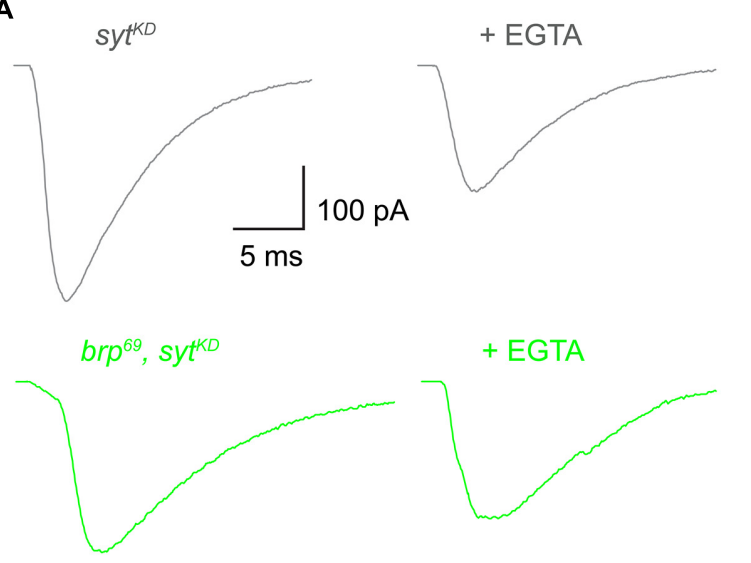

B

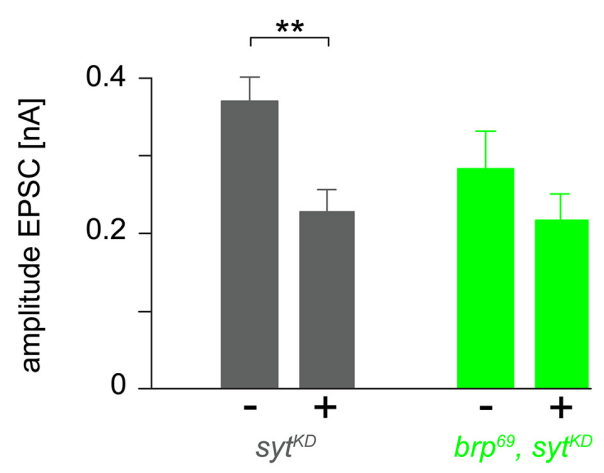

FIGURE 4 | Reduced EGTA sensitivity in brp $^{69}$, syt $^{K D}$. (A) Focally recorded, averaged EPSCs from syt ${ }^{K D}$ (gray) and brp ${ }^{69}$, syt $^{K D}$ (light green) NMJs under control conditions and after incubation with $100 \mu \mathrm{M}$ EGTA-AM for 10 min (+EGTA). (B) Summary bar graph of EPSC amplitudes without (-) or with (+) EGTA-AM in both genotypes. in proximal boutons $(16.5 \pm 0.8,9.8 \pm 0.7,9.6 \pm 0.8, p<0.001$, $n=115$ (1), 70 (2), 70 (3), Figure 5B). In addition, distal type Ib boutons were largest along the bouton chain $\left(10.6 \pm 0.5 \mu \mathrm{m}^{2}\right.$, $\left.6.9 \pm 0.5 \mu \mathrm{m}^{2}, 6.5 \pm 0.5 \mu \mathrm{m}^{2}, p<0.001\right)$. Interestingly, this gradient was not found in type Is boutons regarding both AZ number $[5.5 \pm 0.4,5.5 \pm 0.3,4.6 \pm 0.3, p>0.05, n=73$ (1), 73 (2), 73 (3)] and bouton area $\left(3.0 \pm 0.2 \mu \mathrm{m}^{2}, 3.1 \pm 0.2 \mu \mathrm{m}^{2}, 3.3 \pm 0.2 \mu \mathrm{m}^{2}\right.$, $p>0.05$, Figure 5C). Our data are in line with previous work analyzing functional (Guerrero et al., 2005; Peled and Isacoff, 2011) and structural properties (Ehmann et al., 2014) of the NMJ. Furthermore, the number of AZs per bouton was decreased at rab3 $3^{r u p}$ NMJs compared to wt (Figures 5D-F). This matches earlier work, showing that Rab3 controls the distribution of Brp at the NMJ with decreased $A Z$ numbers per NMJ and increased Brp levels per AZ in rab3 ${ }^{\text {rup }}$ (Graf et al., 2009). However, the structural gradient regarding AZ number per bouton and bouton size was still present in rab3 $3^{\text {rup }}$ type $\mathrm{Ib}$ axons [Brp: $7.0 \pm 0.3,4.1 \pm 0.3,4.2 \pm 0.3$; area: $10.5 \pm 0.5 \mu \mathrm{m}^{2}, 6.9 \pm 0.5 \mu \mathrm{m}^{2}, 7.5 \pm 0.5 \mu \mathrm{m}^{2}, p<0.001$, respectively, $n=117$ (1), 57 (2), 57 (3), Figure 5E]. Thus, a structural gradient is present along the MN6/7b-Ib motor neuron with larger distal than proximal type Ib boutons. In addition, Brp is unevenly distributed in type Ib boutons of wt and rab3 ${ }^{\text {rup }}$ NMJs.

\section{SYT AND BRP ARE ESSENTIAL FOR FUNCTIONAL PRESYNAPTIC DIFFERENTIATION}

We used focal electrodes as these can be selectively placed on a subset of presynaptic boutons to improve spatial resolution of synaptic measurements. Boutons were visualized by GFP-expression (Pawlu et al., 2004) and postsynaptic currents of proximal and distal type $\mathrm{Ib}$ boutons were measured in response to low-frequency nerve stimulation in $0.5 \mathrm{mM}\left[\mathrm{Ca}^{2+}\right]_{\mathrm{Ex}}$ (Figure 6). Distal boutons of wt NMJs showed larger EPSC amplitudes and shorter rise times than proximal boutons (amplitude: $1.4 \pm 0.1 \mathrm{nA}$ and $1.0 \pm 0.1 \mathrm{nA}$, $p=0.005 ; \mathrm{rt}: 1.1 \pm 0.07 \mathrm{~ms}$ and $1.3 \pm 0.07 \mathrm{~ms}, p=0.032, n=24$, and 33, Figure 6B). In contrast, at both $b r p^{69}$ and $s y t^{K D}$ NMJs amplitude and kinetics of postsynaptic currents were comparable in distal and proximal boutons $\left(n=10\right.$ and 11 for $b r p^{69}$ and 11 and 13 for $s y t^{K D}$, Figure 6B). These results reveal that Brp and Syt are both essential for the functional differentiation of the NMJ.

\section{SYT AND BRP INTERACT GENETICALLY}

To test for a genetic interaction between Brp and Syt we analyzed non-allelic non-complementation (Yook et al., 2001). This genetic strategy tests for the ability of two recessive mutations to complement one another for a specific phenotype. We studied heterozygous animals carrying either one copy of the syt null allele $S y t^{A D 4}$ (DiAntonio et al., 1993) or the $\operatorname{brp}$ null allele $b r p^{69}$ (Kittel et al., 2006) and trans-heterozygous animals carrying both. We analyzed the structural gradient along the MN6/7b-Ib motor axon regarding AZ number and bouton dimensions (Figure 7). Whereas in heterozygous animals distal boutons contained more Brp puncta than proximal boutons ( $p<0.001, n=95$ (1), 72 (2), 72 (3) for $s y t^{A D 4} ; p<0.001, n=51$ (1), 40 (2), 40 (3) for $b r p^{69}$ ), this gradient was lost in trans-heterozygous animals $(9.8 \pm 0.5$, $10.0 \pm 0.4,11.0 \pm 0.5, p>0.05, n=81$ (1), 81 (2), 81 (3), Figure 7A). Interestingly, distal boutons of trans-heterozygous animals were still largest along the bouton chain $\left(7.3 \pm 0.4 \mu \mathrm{m}^{2}\right.$, 


\section{A}

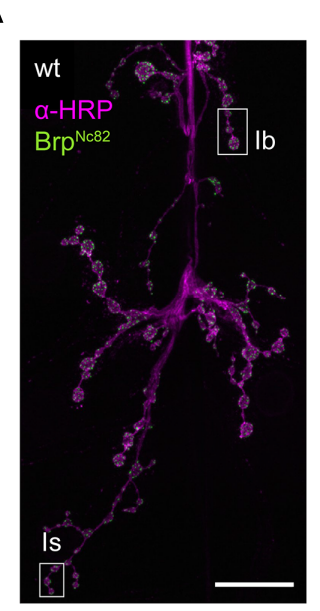

D

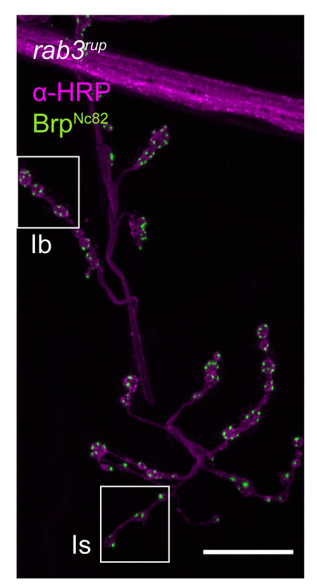

B

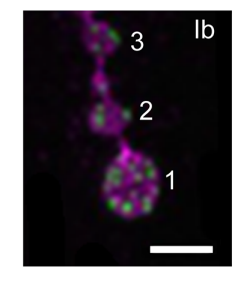

C

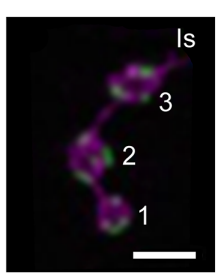

E

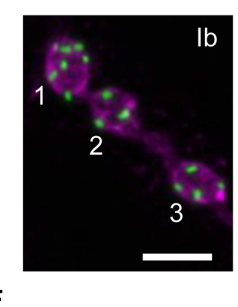

$\mathbf{F}$

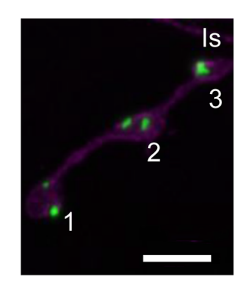

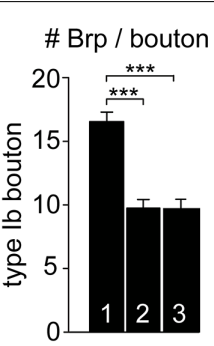
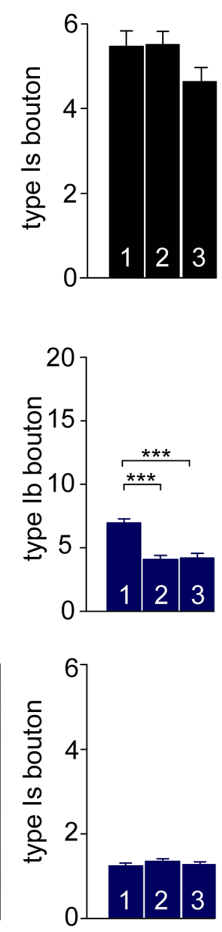
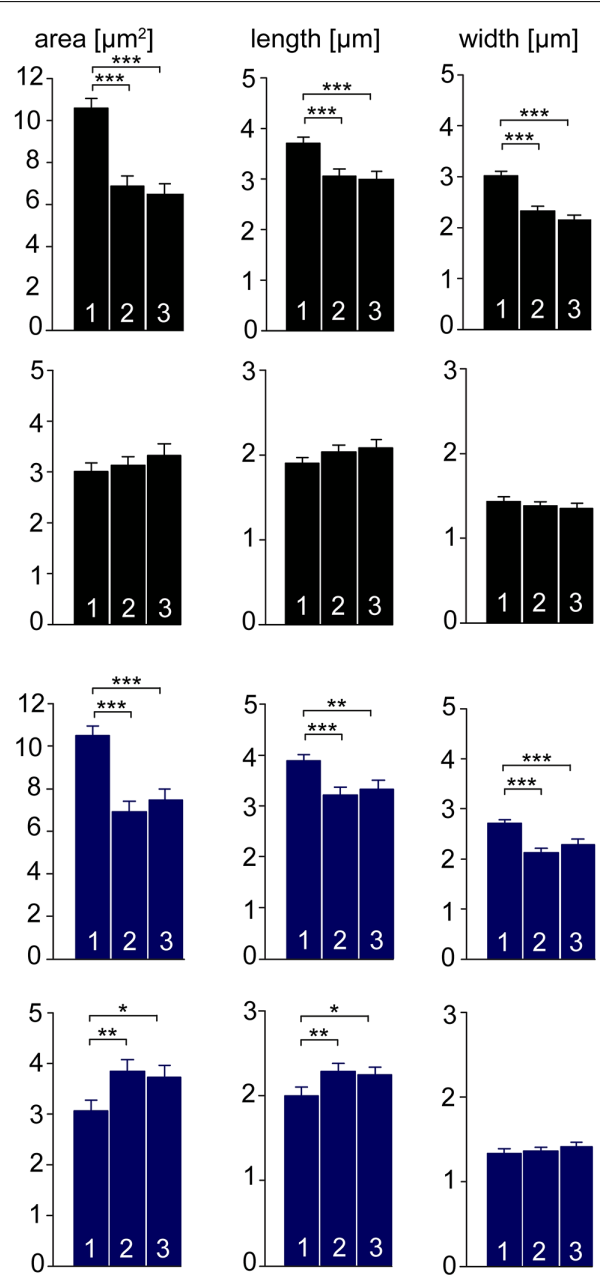

No. of bouton from end

FIGURE 5 | Type lb and type Is bouton morphometry in wt and rab3 ${ }^{\text {rup }}$. (A) Drosophila wt NMJ stained with $\alpha$-HRP against neuronal membranes (magenta) and $\mathrm{mAb} \mathrm{Brp}^{N c 82}$ against Brp (green). White boxes indicate terminal three boutons of type $\mathrm{Ib}$ and type Is branches. (B,C) Magnification of boxed regions in (A). Summary bar graphs for the number of Brp puncta per bouton and bouton area, length and width of the terminal three boutons. (D) Drosophila rab3rup NMJ as in (A). Neurite passing by the $\mathrm{NMJ}$ at the top. (E,F) Magnification of boxed regions in (D) show the terminal three boutons of respective type $\mathrm{Ib}$ and type Is endings. Summary bar graphs for the number of Brp puncta per bouton and area, length and width of the terminal three boutons. Scale bars $=20 \mu \mathrm{m}(\mathbf{A}, \mathbf{D})$ and $5 \mu \mathrm{m}$ $(\mathrm{B}, \mathrm{C}, \mathrm{E}, \mathrm{F})$.
$5.5 \pm 0.3 \mu \mathrm{m}^{2}, 5.8 \pm 0.3 \mu \mathrm{m}^{2}, p=0.003$ and 0.014 for (2) and (3), Figure 7B). We conclude that regarding AZ distribution both mutations fail to complement one another, suggesting a direct interaction of Brp and Syt or an action of the two proteins in the same functional pathway.

\section{SYT GUIDES AZ DISTRIBUTION}

To further investigate the impact of Brp and Syt on maintaining the structural gradient we again performed immunostainings (Figure 8). At $b r p^{69} \mathrm{NMJs}$, bouton area, length, and width were larger for distal than for proximal type Ib boutons (data not shown). Analysis of $s y t^{K D}$ NMJs showed profound alterations of synaptic morphology regarding Brp distribution and bouton size. Whereas the number of Brp positive AZs per NMJ was slightly decreased compared to wt $(724 \pm 38$ and $861 \pm 41, p=0.019$, $n=16$ NMJs each), AZ numbers per Ib bouton were reduced to about a quarter. Furthermore, Brp was distributed homogeneously along the MN6/7b-Ib motor neuron and spatial dimensions of type Ib boutons were similar for all locations along the motoneuron $[p>0.05, n=125$ (1), 109 (2), 109 (3), Figures 8B,C]. Compared to wt, bouton size was reduced dramatically. In addition, analysis of the structural gradient in combined rab3 $3^{r u p}, s y t^{K D}$ animals revealed that Syt knock-down also decreases the structural differentation at rab3 ${ }^{\text {rup }}$ NMJs (data not shown). These data demonstrate that Syt is essential for the structural differentation of the NMJ both in wt and rab3 $3^{r u p}$.

\section{SYT INFLUENCES ORGANIZATION AND NUMBER OF BRP PROTEINS AT INDIVIDUAL AZs}

In a final set of experiments we employed $d S T O R M$ to image glutamatergic boutons. This localization microscopy technique substantially increases spatial resolution compared to conventional 

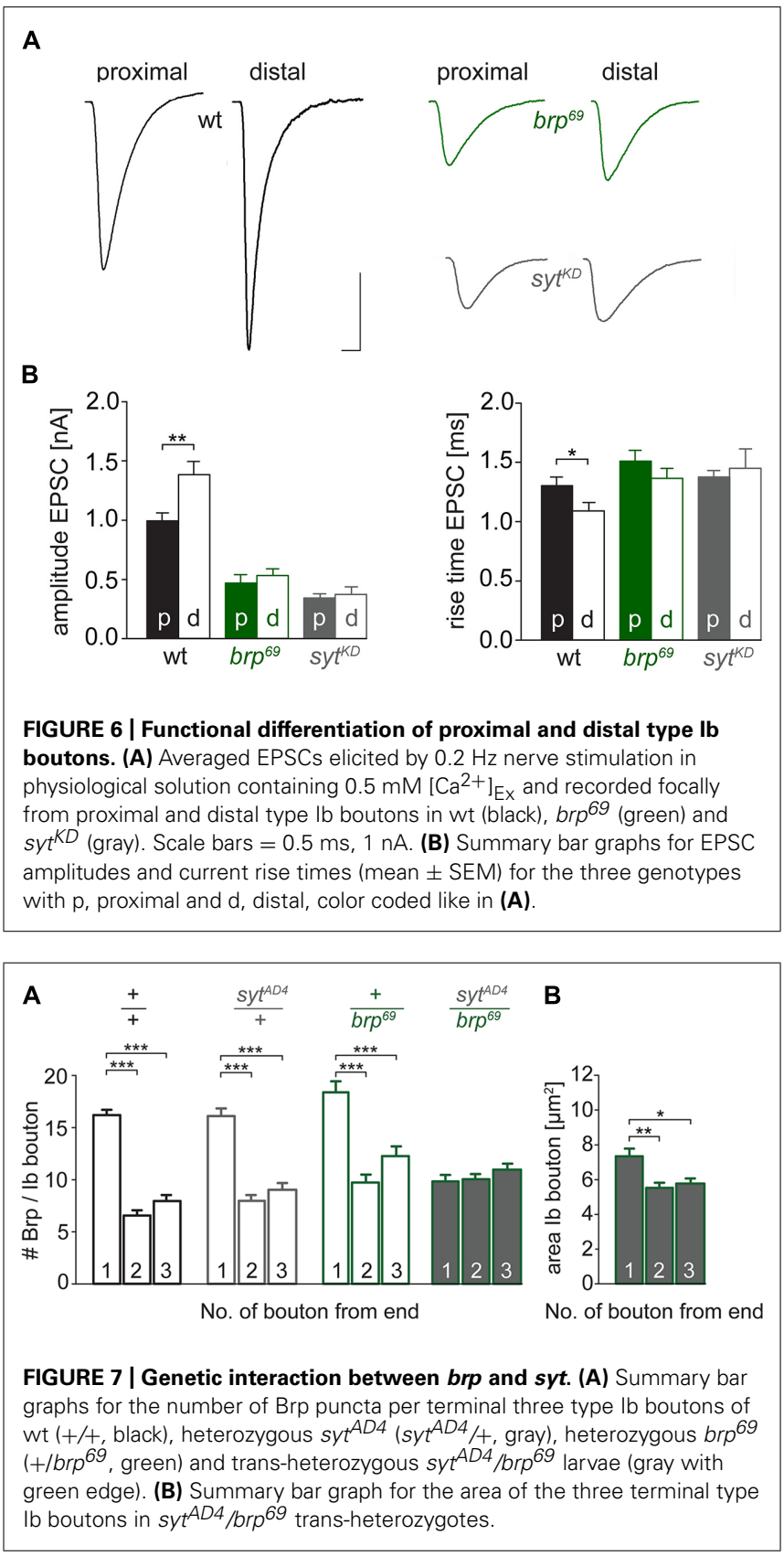

fluorescence light microscopy (Heilemann et al., 2008; van de Linde etal., 2011) and can provide quantitative insight into the nanoscopic organization of presynaptic AZs (Sauer, 2013; Ehmann et al., 2014, 2015). dSTORM resolved the substructural arrangement of indiviudal Brp localizations into multiple clusters within single AZs, which correspond to diffraction-limited Brp puncta in confocal images. We analyzed AZs in the distal six type $\mathrm{Ib}$ boutons of MN6/7b-Ib motor neurons in wt and $s y t^{K D}$ (Figure 9). Interestingly, syt ${ }^{K D} \mathrm{AZs}$ were larger than their wt counterparts $\left(0.079 \pm 0.003 \mu \mathrm{m}^{2}\right.$ and $0.069 \pm 0.002 \mu \mathrm{m}^{2}$, $p=0.003, n=300$, and $468 \mathrm{AZs}$, Figure 9D) and contained more localizations $(710 \pm 30$ and $590 \pm 19, p=0.003$, Figure 9E), which reflects an increased number of Brp protein copies (Ehmann etal., 2014). Furthermore, we observed that syt ${ }^{K D}$ AZs contained a similar number of Brp localizations irrespective of bouton order (Figure 9F, Spearman correlation coefficient $r=-0.169, p<0.001$ for wt and $r=0.014$, $p>0.05$ for $s y t^{K D}$ indicates a moderate negative correlation for wt and no correlation for $s y t^{K D}$ ). Thus, Syt influences the arrangement of Brp at the AZ. Previous work showed that the number of Brp localizations per wt AZ is higher in distal than in proximal type Ib boutons (Ehmann et al., 2014). This is consistent with the electrophysiological and structural data presented here.

\section{DISCUSSION}

While differentiation of presynaptic terminals was initially described more than 50 years ago and has since been studied extensively in various organisms, its mechanisms remain poorly understood (Katz, 1936; Hoyle and Wiersma, 1958; Reyes et al., 1998; reviewed in Atwood and Karunanithi, 2002). In view of the enormous complexity of the relevant molecular mechanisms (Südhof, 2012), the genetically and experimentally accessible NMJ of Drosophila melanogaster provides advantageous features for studying a glutamatergic synaptic system. Type Is- and Ib-boutons of the NMJ exhibit distinct functional properties (Kurdyak et al., 1994; Pawlu et al., 2004), show differences in vesicle size (Karunanithi et al., 2002) and in the amount of Brp molecules per AZ (Ehmann et al., 2014).

Here we show branch-specific differentiation in the MN6/7b-Ib motoneuron regarding structure and function. Distal type Ib boutons are larger than proximal ones, have more Brp positive AZs and show larger and faster postsynaptic responses (Figures $\mathbf{5}$ and $\mathbf{6}$ ). Consistent with these findings, AZs of distal type Ib boutons are larger and possess more Brp molecules per AZ (Ehmann et al., 2014). Presynaptic differentiation is impaired by disrupting either Brp or Syt function (Figures 6, 8, and 9). Postsynaptic responses of proximal and distal boutons in $b r p^{69}$ and $s y t^{K D}$ are comparable and the structural gradient in bouton size, AZs per bouton and AZ size is absent in $s y t^{K D}$. Moreover, genetic evidence suggests that $\mathrm{Brp}$ and Syt act in the same functional pathway to mediate structural heterogeneity (Figure 7). Structural and functional presynaptic differentiation thus clearly requires the concerted action of Brp and Syt.

Interestingly, we obtained consistently lower values for $\mathrm{AZ}$ size and Brp counts per AZ than a recent previous investigation using dSTORM (Ehmann etal., 2014). In the present study, we raised both mutant and control animals at $29^{\circ} \mathrm{C}$ to ensure efficient RNA-mediated $s y t^{K D}$ (expression via the GAL4UAS system is temperature-dependent), whereas Ehmann et al. (2014) raised larvae at $25^{\circ} \mathrm{C}$. Since higher temperature accelerates the development of Drosophila, enlarges presynaptic arborizations and increases the number of AZs per NMJ (Ashburner, 1989; Sigrist etal., 2003), it is conceivable that temperaturedependent plasticity also affects molecular organization at the level of individual AZs.

The molecular mechanisms controlling size, structure and distribution of AZs are complex. Several years ago, the vesicle protein Rab3 was identified as an important regulatory factor of AZ size and distribution (Graf etal., 2009). Rab-proteins 
A

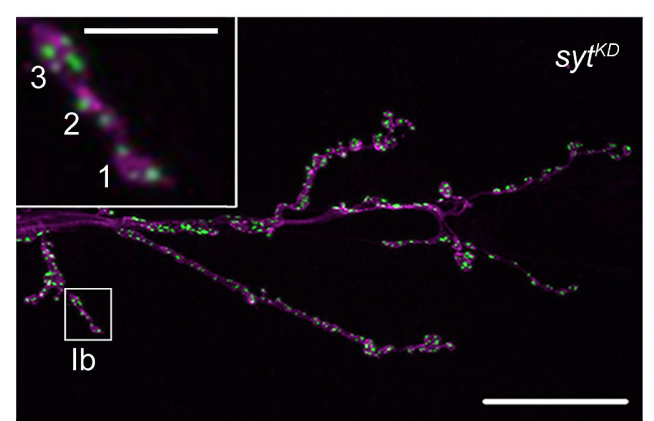

C

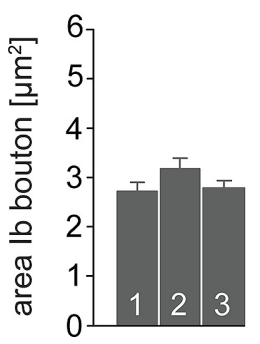

B
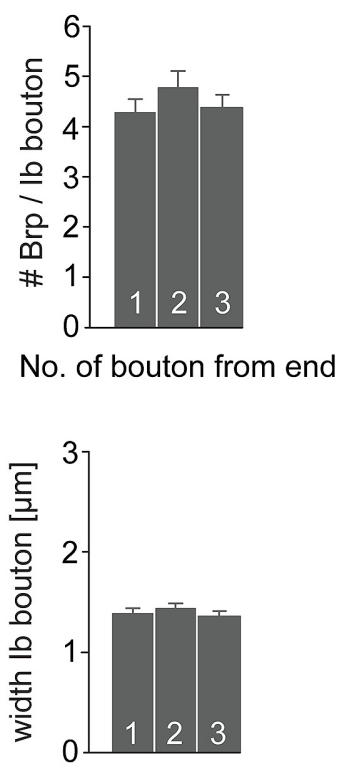

No. of bouton from end

FIGURE 8 |Type lb bouton morphometry in syt ${ }^{K \boldsymbol{D}}$. (A) syt ${ }^{K D} \mathrm{NMJ}$ stained with $\alpha$-HRP against neuronal membranes (magenta) and mAb Brp ${ }^{N c 82}$ (green). Higher magnification of type lb ending (white box) showing the three terminal boutons. Scale bars $=20 \mu \mathrm{m}, 2 \mu \mathrm{m}$ (inset). (B) Summary bar graph for the number of Brp puncta per terminal three type lb boutons in $s y t^{K D}$ and (C) bouton area, length, and width. are key organizers of vesicle trafficking (Harris and Littleton, 2011). Measurements with genetically encoded postsynaptic calcium sensor showed comparable calcium signals in proximal and distal type Ib boutons at rab3 ${ }^{\text {rup }}$ NMJs (Peled and Isacoff, 2011). Here, we found a gradient in rab3 ${ }^{\text {rup }}$ animals in the number of Brp positive AZs along the MN6/7b-Ib motoneuron (Figure 5), unlike in $s y t^{K D}$ (Figure 8). Whereas at rab3 ${ }^{r u p}$ NMJs the overall number of AZs is reduced dramatically (Graf et al., $2009)$, this reduction is moderate at $s y t^{K D}$ NMJs $(724 \pm 38$ and $861 \pm 41$, see Results). However, at both rab3 ${ }^{\text {rup }}$ and $s y t^{K D}$ type Ib branches, the number of Brp proteins per AZ is increased strongly and moderately, respectively (Ehmann et al., 2014; Figure 9). In contrast to rab3 ${ }^{\text {rup }}$ synapses, $s y t^{K D}$ decreases bouton size. Smaller boutons were reported for $s y t^{A D 4}$ and linked to defects in endocytosis (Dickman etal., 2006). We found that brp and syt interact genetically regarding $\mathrm{AZ}$ number per bouton, but not area of boutons (Figure 7), which suggests that the effects of Syt on bouton size and AZ-differentiation are not strictly linked.

The pronounced presynaptic structural alterations after $s y t^{K D}$ are puzzling. Syt is one of the best-studied synaptic proteins. However, its function has mainly been discussed without considering AZ-differentiation. After $s y t^{K D}$, evoked release was reduced by a factor of 4-5 compared to wt in our focal recordings (Figure 6). However, the number of Brp spots in terminal boutons was also reduced by a factor of 4-5 after $s y t^{K D}$ (Figure 8). Is release probability per $\mathrm{AZ}$ in distal boutons following $s y t^{K D}$ therefore similar to wt? More work perhaps combining optical release sensors, focal recordings and subsequent immunostainings will be necessary to clarify this issue. The present study highlights how interpretations of synaptic function and differentiation profit from electrophysiological recording techniques with improved spatial resolution (focal vs. TEVC). Functional sampling of synaptic subsets appears absolutely necessary when considering the significant differentiation at the structural level. Either way, linking structure and function at one and the same AZ is fundamentally important for a comprehensive mechanistic interpretation (Bailey and Chen, 1983; Wojtowicz et al., 1994).

Our electrophysiological data suggest that Syt protracts release at AZs lacking Brp. In this study, we used $s y t^{K D}$ to reduce the protein level in presynpatic terminals (Figure 2). While we assume there are normally more than 10 Syt molecules on each vesicle in our preparation (Takamori et al., 2006), it is unclear whether $s y t^{K D}$ leads to a reduction in the average number of Syt proteins per vesicle or a reduction in the number of Syt positive vesicles with those remaining possessing a full complement of Syt copies. This is relevant in the context of the molecular interpretation of our results. For example, ring-like oligomerization of Syt's cytosolic C2-domains, which prevents release in the absence of calcium, requires a certain copy number (Wang etal., 2014). Furthermore, quantitative information on Syt's partner molecules, such as Complexin and SNAREproteins, will be required for a mechanistic interpretation down to the level of stochiometric interactions (Mohrmann et al., 2010; Cho etal., 2014). Imaging techniques such as dSTORM can be used to quantify the molecular organization of AZs (Sauer, 2013; Ehmann etal., 2015) and will be necessary to 
A
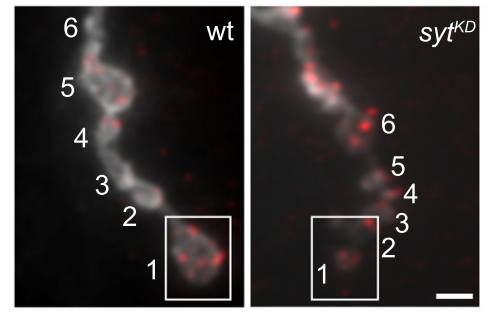

B

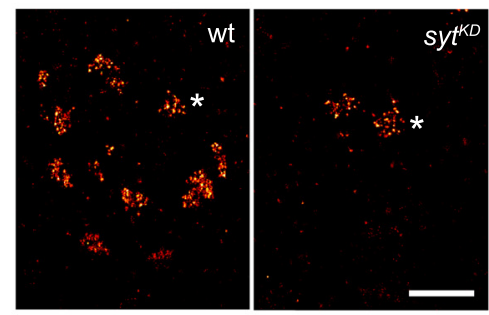

C

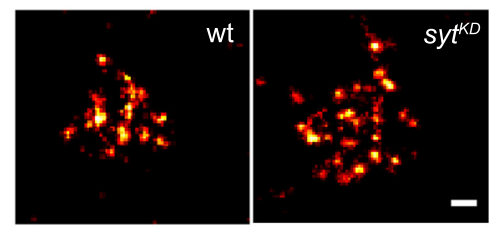

D

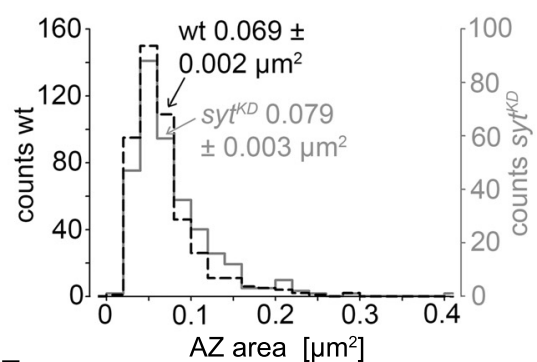

E

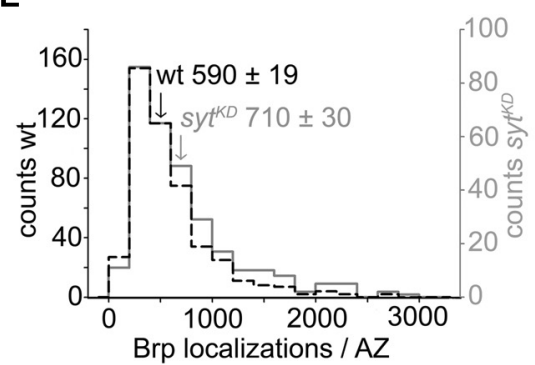

F

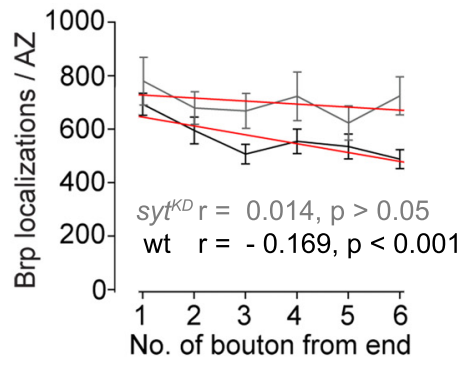

$A Z$ areas and (E) distribution of Brp localizations per $A Z$ are shifted to larger values in $s y t^{K D}$ (gray). Arrows indicate respective mean $\pm S E M$ values. (F) Linear fits (red) of mean \pm SEM Brp localizations per AZ in the terminal six type $\mathrm{lb}$ boutons had slopes of -33.2 (wt) and -11.2 (syt ${ }^{K D}$ ) (non-parametric Spearman correlation coefficient wt: $r=-0.169, p<0.001$; syt ${ }^{K D}: r=0.014$, $p>0.05)$. Scale bars $=2 \mu \mathrm{m}$ (A), $1 \mu \mathrm{m}$ (B), $100 \mathrm{~nm}$ (C).

clarify the so far insufficiently understood kinetic release parameters. In this context, interpretations may well have to take into account the existence of alternative sensors (Walter et al., 2011).

Syt contributes to vesicle docking at the AZ, vesicle positioning within the AZ, clamping and triggering release from the AZ (Walter et al., 2011). While specific amino acids of Syt have been put in connection with certain subsets of these features (e.g., Young and Neher, 2009), it remains unclear which specific functional roles and molecular domains of Syt are responsible for interactions with Brp and structural synaptic specialization. Intriguingly, both Syt and Rab3 are involved in vesicle trafficking and participate in the structural differentiation of AZs. Our work supports the notion that organization of the synaptic vesicle cycle and AZ structure are causally linked.

\section{AUTHOR CONTRIBUTIONS}

Mila M. Paul, Martin Pauli, Nadine Ehmann, Stefan Hallermann, Markus Sauer, and Manfred Heckmann performed experiments.
Mila M. Paul, Stefan Hallermann, Robert J. Kittel, and Manfred Heckmann analyzed the data. Robert J. Kittel and Manfred Heckmann conceived the project and coordinated the study. Mila M. Paul, Robert J. Kittel, and Manfred Heckmann wrote the manuscript with assistance from all co-authors.

\section{ACKNOWLEDGMENTS}

This work was supported by grants from the German Research Foundation (DFG) to Robert J. Kittel (KI 1460/1-1 and SFB 1047/A05), from the IZKF Würzburg to Manfred Heckmann (N229), a BMBF grant to Markus Sauer (133N12781) and a GSLS-fellowship from the University of Würzburg to Nadine Ehmann. The authors thank N. Reist for the $\alpha$-Dsyt-CL1 antibody, D. Ljaschenko for support in the intitial phase of the study and F. Köhler, T. Martini, M. Oppmann, and C. Wirth for technical assistance.

\section{REFERENCES}

Ashburner, M. (1989). Drosophila: A Laboratory Handbook. Cold Spring Harbor, NY: Cold Spring Harbor Laboratory Press. 
Atwood, H. L., Govind, C. K., and Wu, C. F. (1993). Differential ultrastructure of synaptic terminals on ventral longitudinal abdominal muscles in Drosophila larvae. J. Neurobiol. 24, 1008-1024. doi: 10.1002/neu.480240803

Atwood, H. L., and Karunanithi, S. (2002). Diversification of synaptic strength: presynaptic elements. Nat. Rev. Neurosci. 3, 497-516. doi: 10.1038/nrn876

Bailey, C. H., and Chen, M. (1983). Morphological basis of long-term habituation and sensitization in Aplysia. Science 220, 91-93. doi: 10.1126/science.6828885

Barrett, E. F., and Stevens, C. F. (1972). The kinetics of transmitter release at the frog neuromuscular junction. J. Physiol. 227, 691-708. doi: 10.1113/jphysiol.1972.sp010054

Brand, A. H., and Perrimon, N. (1993). Targeted gene expression as a means of altering cell fates and generating dominant phenotypes. Development 118 , 401-415.

Brose, N., Petrenko, A. G., Südhof, T. C., and Jahn, R. (2002). Synaptotagmin: a calcium sensor on the synaptic vesicle surface. Science 256, 1021-1025. doi: $10.1126 /$ science. 1589771

Cho, R. W., Kümmel, D., Li, F., Baguley, S. W., Coleman, J., and Rothman, J. E. et al. (2014). Genetic analysis of the Complexin trans-clamping model for crosslinking SNARE complexes in vivo. Proc. Natl. Acad. Sci. U.S.A. 111, 10317-10322. doi: 10.1073/pnas.1409311111

Datyner, N. B., and Gage, P. W. (1980). Phasic secretion of acetylcholine at a mammalian neuromuscular junction. J. Physiol. 303, 299-314. doi: 10.1113/jphysiol.1980.sp013286

DeBello, W. M., Betz, H., and Augustine, G. J. (1993). Synaptotagmin and neurotransmitter release. Cell 74, 947-950. doi: 10.1016/0092-8674(93)90716-4

DiAntonio, A., Parfitt, K. D., and Schwarz, T. L. (1993). Synaptic transmission persists in synaptotagmin mutants of Drosophila. Cell 73, 1281-1290. doi: 10.1016/0092-8674(93)90356-U

Dickman, D. K., Lu, Z., Meinertzhagen, I. A., and Schwarz, T. L. (2006). Altered synaptic development and active zone spacing in endocytosis mutants. Curr. Biol. 16, 591-598. doi: 10.1016/j.cub.2006.02.058

Dudel, J. (1965). Potential changes in the crayfish motor nerve terminal during repetitive stimulation. Pflügers Arch. Gesamte Physiol. Menschen Tiere 282, 323 337. doi: 10.1007/BF00412507

Eggermann, E., Bucurenciu, I., Goswami, S. P., and Jonas, P. (2011). Nanodomain coupling between $\mathrm{Ca} 2+$ channels and sensors of exocytosis at fast mammalian synapses. Nat. Rev. Neurosci. 13, 7-21. doi: 10.1038/nrn3125

Ehmann, N., Sauer, M., and Kittel, R. J. (2015). Super-resolution microscopy of the synaptic active zone. Front. Cell. Neurosci. 9:7. doi: 10.3389/fncel.2015. 00007

Ehmann, N., van de Linde, S., Alon, A., Ljaschenko, D., Keung, X. Z., Holm, T., et al. (2014). Quantitative super-resolution imaging of Bruchpilot distinguishes active zone states. Nat. Commun. 5, 4650. doi: 10.1038/ncomms5650

Geppert, M., Goda, Y., Hammer, R. E., Li, C., Rosahl, T. W., Stevens, C. F., et al. (1994). Synaptotagmin I: a major Ca2+ sensor for transmitter release at a central synapse. Cell 79, 717-727. doi: 10.1016/0092-8674(94)90556-8

Graf, E. R., Daniels, R. W., Burgess, R. W., Schwarz, T. L., and DiAntonio, A. (2009). Rab3 dynamically controls protein composition at active zones. Neuron 64, 663-677. doi: 10.1016/j.neuron.2009.11.002

Guerrero, G., Reiff, D. F., Agarwal, G., Ball, R. W., Borst, A., Goodman, C. S., et al. (2005). Heterogeneity in synaptic transmission along a Drosophila larval motor axon. Nat. Neurosci. 8, 1188-1196. doi: 10.1038/nn1526

Harris, K. P., and Littleton, J. T. (2011). Vesicle trafficking: a Rab family profile. Curr. Biol. 21, 841-843. doi: 10.1016/j.cub.2011.08.061

Heilemann, M., van de Linde, S., Schüttpelz, M., Kasper, R., Seefeldt, B., Mukherjee, A., et al. (2008). Subdiffraction-resolution fluorescence imaging with conventional fluorescent probes. Angew. Chem. Int. Ed. Engl. 47, 6172-6176. doi: 10.1002/anie. 200802376

Hoang, B., and Chiba, A. (2001). Single-cell analysis of Drosophila larval neuromuscular synapses. Dev. Biol. 229, 55-70. doi: 10.1006/dbio.2000.9983

Hoyle, G., and Wiersma, C. A. (1958). Excitation at neuromuscular junctions in Crustacea. J. Physiol. 143, 403-425. doi: 10.1113/jphysiol.1958.sp006068

Jan, L. Y., and Jan, Y. N. (1982). Antibodies to horseradish peroxidase as specific neuronal markers in Drosophila and in grasshopper embryos. Proc. Natl. Acad. Sci. U.S.A. 79, 2700-2704. doi: 10.1073/pnas.79.8.2700

Karunanithi, S., Marin, L., Wong, K., and Atwood, H. L. (2002). Quantal size and variation determined by vesicle size in normal and mutant Drosophila glutamatergic synapses. J. Neurosci. 22, 10267-10276.
Katz, B. (1936). Neuro-muscular transmission in crabs. J. Physiol. 87, 199-221. doi: 10.1113/jphysiol.1936.sp003401

Kittel, R. J., Wichmann, C., Rasse, T. M., Fouquet, W., Schmidt, M., Schmid, A., et al. (2006). Bruchpilot promotes active zone assembly, Ca2+ channel clustering, and vesicle release. Science 312, 1051-1054. doi: 10.1126/science. 1126308

Kurdyak, P., Atwood, H. L., Stewart, B. A., and Wu, C. F. (1994). Differential physiology and morphology of motor axons to ventral longitudinal muscles in larval Drosophila. J. Comp. Neurol. 350, 463-472. doi: 10.1002/cne. 903500310

Littleton, J. T., Stern, M., Schulze, K., Perin, M., and Bellen, H. J. (1993). Mutational analysis of Drosophila synaptotagmin demonstrates its essential role in Ca2+-activated neurotransmitter release. Cell 74, 1125-1134. doi: 10.1016/0092-8674(93)90733-7

Lnenicka, G. A., and Keshishian, H. (2000). Identified motor terminals in Drosophila larvae show distinct differences in morphology and physiology. J. Neurobiol. 43, 186-197. doi: 10.1002/(SICI) 1097-4695(200005)43:2<186::AIDNEU8 $>3.0 . \mathrm{CO} ; 2-\mathrm{N}$

Mackler, J. M., Drummond, J. A., Loewen, C. A., Robinson, I. M., and Reist, N. E. (2002). The C2B Ca2+-binding motif of synaptotagmin is required for synaptic transmission in vivo. Nature 418, 340-344. doi: 10.1038/nature00846

Matz, J., Gilyan, A., Kolar, A., McCarvill, T., and Krueger, S. R. (2010). Rapid structural alterations of the active zone lead to sustained changes in neurotransmitter release. Proc. Natl. Acad. Sci. U.S.A. 107, 8836-8841. doi: 10.1073/pnas.0906087107

Maximov, A., and Südhof, T. C. (2005). Autonomous function of synaptotagmin 1 in triggering synchronous release independent of asynchronous release. Neuron 48, 547-554. doi: 10.1016/j.neuron.2005.09.006

Mohrmann, R., de Wit, H., Verhage, M., Neher, E., and Sørensen, J. B. (2010). Fast vesicle fusion in living cells requires at least three SNARE complexes. Science 330, 502-505. doi: 10.1126/science.1193134

Müller, M., Liu, K. S., Sigrist, S. J., and Davis, G. W. (2012). RIM controls homeostatic plasticity through modulation of the readily-releasable vesicle pool. J. Neurosci. 32, 16574-16585. doi: 10.1523/JNEUROSCI.0981-12.2012

Neher, E. (1998). Vesicle pools and Ca2+ microdomains: new tools for understanding their roles in neurotransmitter release. Neuron 20, 389-399. doi: 10.1016/S0896-6273(00)80983-6

Neher, E. (2010). What is rate-limiting during sustained synaptic activity: vesicle supply or the availability of release sites. Front. Synaptic Neurosci. 2:144. doi: 10.3389/fnsyn.2010.00144

Pawlu, C., DiAntonio, A., and Heckmann, M. (2004). Postfusional control of quantal current shape. Neuron 42, 607-618. doi: 10.1016/S0896-6273(04)00269-7

Peled, E. S., and Isacoff, E. Y. (2011). Optical quantal analysis of synaptic transmission in wild-type and rab3-mutant Drosophila motor axons. Nat. Neurosci. 14, 519-526. doi: 10.1038/nn.2767

Peled, E. S., Newman, Z. L., and Isacoff, E. Y. (2014). Evoked and spontaneous transmission favored by distinct sets of synapses. Curr. Biol. 24, 484-493. doi: 10.1016/j.cub.2014.01.022

Reyes, A., Lujan, R., Rozov, A., Burnashev, N., Somogyi, P., and Sakmann, B. (1998). Target-cell-specific facilitation and depression in neocortical circuits. Nat. Neurosci. 1, 279-285. doi: 10.1038/1092

Sauer, M. (2013). Localization microscopy coming of age: from concepts to biological impact. J. Cell Sci. 126, 3505-3513. doi: 10.1242/jcs.123612

Schmidt, H., Brachtendorf, S., Arendt, O., Hallermann, S., Ishiyama, S., Bornschein, G., et al. (2013). Nanodomain coupling at an excitatory cortical synapse. Curr. Biol. 23, 244-249. doi: 10.1016/j.cub.2012.12.007

Sigrist, S. J., Reiff, D. F., Thiel, P. R., Steinert, J. R., and Schuster, C. M. (2003). Experience-dependent strengthening of Drosophila neuromuscular junctions. J. Neurosci. 23, 6546-6556.

Stewart, B. A., Atwood, H. L., Renger, J. J., Wang, J., and Wu, C. F. (1994). Improved stability of Drosophila larval neuromuscular preparations in haemolymph-like physiological solutions. J. Comp. Physiol. A 175, 179-191. doi: 10.1007/BF00215114

Südhof, T. C. (2012). The presynaptic active zone. Neuron 75, 11-25. doi: 10.1016/j.neuron.2012.06.012

Takamori, S., Holt, M., Stenius, K., Lemke, E. A., Grønborg, M., and Riedel, D. et al. (2006). Molecular anatomy of a trafficking organelle. Cell 127, 831-846. doi: $10.1016 /$ j.cell.2006.10.030 
van de Linde, S., Kasper, R., Heilemann, M., and Sauer, M. (2008). Photoswitching microscopy with standard fluorophores. Appl. Phys. B 93, 725-731. doi: 10.1007/s00340-008-3250-9

van de Linde, S., Löschberger, A., Klein, T., Heidbreder, M., Wolter, S., Heilemann, M., etal. (2011). Direct stochastic optical reconstruction microscopy with standard fluorescent probes. Nat. Protoc. 6, 991-1009. doi: 10.1038/nprot.2011.336

Vyleta, N. P., and Jonas, P. (2014). Loose coupling between Ca2+ channels and release sensors at a plastic hippocampal synapse. Science 343, 665-670. doi: $10.1126 /$ science. 1244811

Wagh, D. A., Rasse, T. M., Asan, E., Hofbauer, A., Schwenkert, I., Dürrbeck, H., et al. (2006). Bruchpilot, a Protein with homology to ELKS/CAST, is required for structural integrity and function of synaptic active zones in Drosophila. Neuron 49, 833-844. doi: 10.1016/j.neuron.2006.02.008

Walter, A. M., Groffen, A. J., Sørensen, J. B., and Verhage, M. (2011). Multiple Ca2+ sensors in secretion: teammates, competitors or autocrats? Trends Neurosci. 34, 487-497. doi: 10.1016/j.tins.2011.07.003

Wang, J., Bello, O., Auclair, S. M., Wang, J., Coleman, J., Pincet, F., et al. (2014). Calcium sensitive ring-like oligomers formed by synaptotagmin. Proc. Natl. Acad. Sci. U.S.A. 111, 13966-13971. doi: 10.1073/pnas.1415849111

Weyhersmüller, A., Hallermann, S., Wagner, N., and Eilers, J. (2011). Rapid active zone remodeling during synaptic plasticity. J. Neurosci. 31, 6041-6052. doi: 10.1523/JNEUROSCI.6698-10.2011

Wojtowicz, J. M., Marin, L., and Atwood, H. L. (1994). Activity-induced changes in synaptic release sites at the crayfish neuromuscular junction. J. Neurosci. 14, 3688-3703.

Wolter, S., Löschberger, A., Holm, T., Aufmkolk, S., Dabauvalle, M. C., van de Linde, S., et al. (2012). rapidSTORM: accurate, fast and open-source software for localization microscopy. Nat. Methods 9, 1040-1041. doi: 10.1038/nmeth.2224
Wolter, S., Schüttpelz, M., Tscherepanow, M., van de Linde, S., Heilemann, M., and Sauer, M. (2010). Real-time computation of subdiffraction-resolution fluorescence images. J. Microsc. 237, 12-22. doi: 10.1111/j.1365-2818.2009. 03287.x

Yook, K. J., Proulx, S. R., and Jorgensen, E. M. (2001). Rules of nonallelic noncomplementation at the synapse in Caenorhabditis elegans. Genetics 158, 209-220.

Young, S. M. Jr., and Neher, E. (2009). Synaptotagmin has an essential function in synaptic vesicle positioning for synchronous release in addition to its role as a calcium sensor. Neuron 63, 482-496. doi: 10.1016/j.neuron.2009. 07.028

Conflict of Interest Statement: The authors declare that the research was conducted in the absence of any commercial or financial relationships that could be construed as a potential conflict of interest.

Received: 28 October 2014; accepted: 16 January 2015; published online: 05 February 2015.

Citation: Paul MM, Pauli M, Ehmann N, Hallermann S, Sauer M, Kittel RJ and Heckmann $M$ (2015) Bruchpilot and Synaptotagmin collaborate to drive rapid glutamate release and active zone differentiation. Front. Cell. Neurosci. 9:29. doi: 10.3389/fncel.2015.00029

This article was submitted to the journal Frontiers in Cellular Neuroscience. Copyright (C) 2015 Paul, Pauli, Ehmann, Hallermann, Sauer, Kittel and Heckmann. This is an open-access article distributed under the terms of the Creative Commons Attribution License (CC BY). The use, distribution or reproduction in other forums is permitted, provided the original author(s) or licensor are credited and that the original publication in this journal is cited, in accordance with accepted academic practice. No use, distribution or reproduction is permitted which does not comply with these terms. 